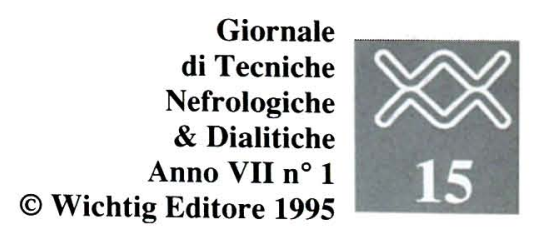

\title{
Rischio di neoplasie nei pazienti in dialisi
}

R. Ragni

\section{Servizio di Nefrologia e Dialisi, Ospedale Martini Nuovo, Torino}

entre nei pazienti immunodepressi con trapianto renale è chiaramente documentato un maggior rischio per alcuni particolari tipi di neoplasie (quali linfomi, neoplasie cutanee, sarcoma di Kaposi), l'aumentata incidenza di tumori insorti nei pazienti in corso di trattamento dialitico, (escludendo quindi i casi di tumore diagnosticati prima dell'immissione in dialisi) è ancora soggetta a discussione.

La prima segnalazione di una maggiore frequenza di neoplasie, con valori sette volte superiori a quelli attesi nella popolazione normale, si deve a Matas e comparve su Lancet nel 1975 (1). Questo dato è stato confermato da diversi articoli in tutto il mondo: Jacobs in Francia (2), ad esempio, Ota in Giappone (3), Bufano in Italia (4), Herr negli Stati Uniti (5). Tutti questi Autori calcolano un "rischio relativo" significativamente elevato, variabile fra $2 / 3$ e 15 , intendendosi come "rischio relativo" il rapporto fra i casi diagnosticati nella popolazione in esame (nel nostro caso i pazienti in dialisi) ed i casi attesi in una popolazione generale di confronto.

Alcuni ricercatori hanno invece osservato una aumentata incidenza riferita solo ad alcuni tipi di neoplasia (rene, vescica, colon, ecc). In contrasto con queste osservazioni altri Autori, in Gran Bretagna (6), e nel Report dell'EDTA del 1987 (7), non hanno trovato differenze significative fra i casi osservati in pa- zienti in dialisi rispetto alla popolazione generale di confronto.

Questa discrepanza di dati può sembrare, a prima vista, incomprensibile, ma un'analisi approfondita di alcuni fattori può rendere conto, almeno in parte, di tali divergenze. Occorre sottolineare infatti: a) assenza di Registri dei Tumori nelle stesse aree di studio e nello stesso periodo di tempo, per cui i raffronti vengono talora riferiti a popolazioni di altre Regioni oppure di altri periodi di tempo; b) differenze di campionamento nei criteri di selezione dei pazienti e di frequenza nei controlli clinici; c) differenti cause ed incidenza di mortalità fra la popolazione uremica e quella generale di controllo; d) nefropatie e patogenesi diversa nelle varie aree geografiche (tipica, ad esempio, la nefropatia dei Balcani oppure la prevalenza di nefropatie IgA nelle popolazioni dell'area mediterranea); e) approcci terapeutici alle malattie renali non univoci e diversa incidenza del trapianto renale.

Pur con queste limitazioni, numerosi elementi (8) sono stati chiamati in causa quali potenziali fattori di incremento del rischio-tumore nei pazienti in dialisi: 1) fattori immunologici. La maggior parte degli Autori è concorde nel considerare che l'immunodepressione costantemente presente nel paziente in dialisi, legata all'uremia di per sé o secondaria all'intervento di tossine uremiche, sia la responsabile primaria dell'elevato rischio di in- fezioni e possa accelerare e/o amplificare il rischio di neoplasie; 2) fattori infettivi. Alcune neoplasie sono state messe in relazione all'insorgenza di infezioni virali, soprattutto croniche: a parte il caso paradigmatico dell'AIDS, esempi classici sono i legami tra virus dell'epatite B e carcinomi epatici oppure tra Papova virus ed alcuni tumori dell'utero; 3) fattori carenziali. Decifit isolati o multipli di zinco, di folati, vitamina $\mathrm{C}$, malnutrizione proteico-calorica possono avere un effetto immunodepressivo; 4) fattori tossici endogeni. Alcuni prodotti del metabolismo azotato potrebbero seguire vie anomale nell'uremia per trasformarsi in amine alifatiche, composti precursori delle nitrosamine, ad azione cancerogena. Inoltre è stato dimostrato che alcuni componenti delle cosiddette medie molecole sono in grado di indurre una diminuzione della chemiotassi leucocitaria. Secondo vari Autori infine, anche il paratormone potrebbe entrare in gioco con differenti meccanismi; 5) fattori tossici esogeni. Numerosi composti potenzialmente tossici possono essere presenti nelle soluzioni per emodialisi quali nitriti, nitrati, cloramine. Lo stesso ossido di etilene, anche se non vi sono evidenze cliniche di carcinogenesi nell'uomo, secondo l'Agenzia Internazionale di Ricerche sul cancro è considerato a rischio negli esperimenti su animali. Infine va ricordato che il sangue dei pazienti è esposto, in corso di dialisi, ai 
composti plastificanti di filtri e linee, quali gli ftalati, di cui è stata descritta un'azione citotossica diretta.

Sulla base di queste osservazioni abbiamo voluto verificare (9) quale fosse la situazione nella Regione Piemonte ove, per altro, viene applicato un criterio di accettazione aperta, basandoci sui dati riportati dal Registro Regionale di Dialisi e Trapianto, aggiornati ogni anno dai singoli centri. Il Registro raccoglie le schede di tutti i pazienti in trattamento sostitutivo dal 1981; nella nostra indagine sono state escluse le neoplasie diagnosticate prima dell'immissione in dialisi e nei primi sei mesi di trattamento. L'analisi delle cause di morte nella nostra popolazone dialitica permette di rilevare come la mortalità per neoplasie abbia raggiunto valori dell' $8 \%$, ma sia percentualmente minore rispetto alla popolazione generale della Regione (33\%), ove occupa il secondo posto; un ruolo preponderante nei pazienti dializzati spetta, invece, alle cause cardiovascolari $(42 \%)$, alla cachessia $(23 \%)$ ed alle infezioni $(9 \%)$. Queste due ultime sono praticamente assenti come causa di morte nella popolazione non uremica.

Suddividendo per fascie di età il pool di pazienti in dialisi, il "rischio relativo" di neoplasie è risultato nettamente maggiore (valore di tre) per il sesso maschile fra 40 e 50 anni, mentre l'incidenza diminuisce significativamente per età superiore ai 70 anni, verosimilmente per il maggior ruolo delle cause di morte competitive con le neoplasie. Inoltre il "rischio relativo" di insorgenza di neoplasie aumenta consensualmente con l'età dialitica: da circa 2.5 nei primi tre anni di dialisi raggiunge il valore di 4 dopo il quarto anno di trattamento. Invece il tipo di trattamento (emodialisi e dialisi peritoneale) non sembra giocare un ruolo significativo.

Le neoplasie non si discostano come distribuzione di frequenza da quanto osservato nella popolazione generale; questo tipo di distribuzione appare in accordo con il riscontro nel paziente uremico di una riduzione a diversi livelli del sistema immunitario di sorveglianza. Si discostano da questa nostra osservazione i dati segnalati in alcune casistiche, che riportano un incremento di particolari neoplasie: ad esempio neoplasie dell'apparato urinario, limitatamente a pazienti con nefropatie da analgesici; altri gruppi hanno segnalato un'incremento esclusivo dei linfomi non Hodgkin. In quest'ottica, particolare attenzione va posta alla possibile degenerazione delle cisti del paziente in trattamento sostitutivo, descritte nella cosiddetta malattia cistica acquisita del rene (ACKD). L'incidenza di tale patologia (le cisti sono multiple e bilaterali, di piccolo diametro, numerose a livello corticale, sempre acquisite quindi assenti all'inizio della dialisi) non va confusa con il rene policistico congenito ed è stata riportata con percentuali variabili dal $40-50 \%$ sino al $90 \%$ dei pazienti in trattamento cronico sostitutivo; la sua frequenza è in relazione alla durata sia dell'uremia, che della dialisi e non è in relazione al tipo di dialisi. Diversi Autori hanno riferito un'alta percetuale di neoplasie a partenza cistica, per cui è raccomandabile una stretta monitorizzazione con ecotomografia e/o con TAC. Per quanto riguarda infine il decorso dei pazienti con neoplasia insorta in corso di dialisi, nella casistica del Registro Regionale del Piemonte la sopravvivenza ad un anno dalla diagnosi è del $48 \% \mathrm{e}$ del $36 \%$ a due anni, sovrapponibile a quanto pubblicato in Europa nel Report dell'EDTA, per cui non vi sono, a nostro avviso, motivi per ritenere la prognosi di questi pazienti tale da non giustificare a priori l'impegno di tutti i presidi terapeutici a disposizione. L'esperienza suggerisce di proseguire il trattamento sostitutivo, modulando tempi e ritmi di dialisi in relazione alle condizioni cliniche del paziente, ma senza tralasciare la terapia oncologica opportuna.

\section{BIBLIOGRAFIA}

1. Matas AJ, Simmons RL, Kjellstrand CM, Buselmeier TJ, Najarian JS. Increased incidence of malignancy during chronic renal failure. Lancet 1975; i: 883-6.

2. Jacobs C, Reach I, Degovlet P. Tumeurs et hemotathies malignes chez les patients traitès par dialyse itèrative. Sem Urol-Nèphrol 1979; 5: 197-211.

3. Ota K, Yamashita N, Suzuki $\mathrm{T}$, Aghishi T. Malignant tumours in dialysis patients: a nationwide survey. Proc EDTA-ERA 1981; 18: 724-9.

4. Bufano G, Biasini A, Magri S, Pecchini F. Indagine italiana sulle neoplasie in corso di dialisi. Min Nefr 1983; 30: 9-12 (suppl).

5. Herr HW, Engen DE, Hostetler J. Malignancy in uremia: dialysis versus transplantation. J Urol 1979; 121: 584-6.

6. Bush A, Gabriel R. Cancer in uremic patients. Clin Nephrol 1984; 22: $77-81$.

7. Brunner FP, Fassbinder W, Broyer M. Combined report on regular dialysis and transplantation in Europe. Proc EDTA-ERA 1987; 24: 10-36.

8. Ragheb NE, Port FK, Schwartz AG. The risk of cancer for patients on dialysis: a review. Seminars in dialysis 1991; 4: 253-7.

9. Ragni R, Piccoli GB. Neoplasie in corso di dialisi. Trattato Italiano di Dialisi. Milano: Wichtig Editore 1990; 8 . 